\title{
Decision making between women and their caregivers during labour ranged from being unilateral to joint and was associated with various emotions
}

Vande Kusse L. Decision making in analyses of women's birth stories. Birth 1999 Mar;26:43-52.

QUESTION: How are decisions made by women and their caregivers during labour and what are the associated patterns of control?

\section{Design}

A reanalysis of 33 birth stories using an exploratory, descriptive design.

\section{Setting}

Midwestern USA.

\section{Participants}

15 women between 18 and 39 years of age who represented a variety of birth experiences. 8 women were primiparous and 7 were multiparous. 12 women were Euro-American and 3 were "women of colour". All women had given birth within the previousm 4 months.

\section{Methods}

Women told their birth stories in any way they wished. Interviews were audiotaped, transcribed verbatim, and analysed using content and thematic analysis. Coders from different disciplines were also recruited to provide fresh perspectives and new interpretations of the data.

\section{Main findings}

4 types of decision making and their associated patterns of control were identified. The first type of decision making was a unilateral decision made by the caregivers that was contested by the women. Women responded with strong, negative emotions (eg, unsettled, sadness, and anger) and either refused care or adapted and submitted to the caregiver's decision. The second type was a unilateral decision made by the caregivers that was uncontested by the women. Women responded by adapting, agreeing, and quietly complying with the decision rather than actively participating in the decision itself. The third type was making no active decision at all and suspending control while waiting to see what would happen as the labour progressed. This type was used in situations with no particular urgency, and women and their caregivers took the time to gather more data, evaluate the situation, and allow the labour to progress. The fourth type described

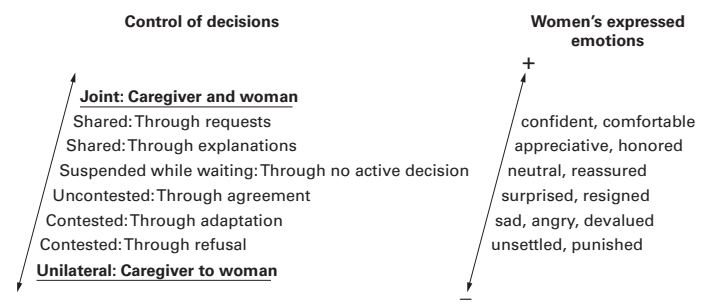

Model of patterns of control and decision-making related to emotions expressed in women's birth stories. Reproduced from Vande Vusse L. Birth 1999;29:43-52 with permission. shared (joint) control between the woman and the caregiver. The decision was made through explanations given by the caregiver to the woman and through requests made by either the woman or caregiver. Women were involved in this type of decision making and could make informed choices, which resulted in positive emotional responses.

\section{Conclusions}

The decision making patterns of women and their caregivers during labour ranged on a continuum from unilateral decisions made by the caregiver to joint decisions made by both the caregiver and the woman. Women responded to these patterns with various emotions ranging from feeling unsettled, punished, sad, angry, and devalued to feeling appreciative, honoured, confident, and comfortable (fig 1).

\section{COMMENTARY}

The study by VandeVusse highlights the important contribution of women in developing effective strategies for the management of childbirth. The qualitative methodology used is an appropriate approach to exploring the question of women's views on decision making during labour. Story telling as an investigative method is well established in the social sciences and its use in healthcare research is growing. ${ }^{1}$ A rigorous approach to data analysis is reported, which supports the identification of 4 types of decision making and their associated patterns of control.

Although the North American setting may limit the applicability of the findings, the study is interesting and may provoke midwives and other healthcare providers to reflect on their own practice. In particular, practitioners may wish to explore ways that women can be involved in decision making at key stages in the birth process so that the birth experience is a positive one. In this study, $64 \%$ of the births were attended by physicians, $24 \%$ by lay midwives, and $9 \%$ by certified nurse midwives. The difference in professional background of birth attendants may have influenced the pattern of control within the decision making process. The author, however, points out that an attempt was made to oversample women who did not have a doctor in attendance in order to maximise the range of reported experiences. It should also be recognised that one third of the sample comprised women who had a caesarean section.

The ethnicity of the women who participated in the study is also relevant to the interpretation of the findings. A wide range of literature supports the diversity of how childbirth is experienced by different ethnic groups, especially between eastern and western cultures, and there is increasing emphasis on the value of consultation between healthcare providers and people from ethnic groups. ${ }^{2}$ Unfortunately, the only information provided for this study is that 12 of the women were of Euro-American descent and 3 were "of colour", and there is no discussion of ethnicity as a factor in the decision making process. In conclusion, this is an interesting and informative study, which may be worthy of replication within a UK context.

$$
\begin{array}{r}
\text { Soraya Meah, RN, RM, MSc } \\
\text { Lecturer in Nursing/Clinical Effectiveness Manager } \\
\text { University of Liverpool } \\
\text { Liverpool, UK }
\end{array}
$$

1 Borges S, Waitzkin H. Women Health 1995;23:29-56.

2 North N. Consumers, service users, or citizens? In: North N, Bradshaw Y, editors. Perspectives in health care. London: Macmillan, 1997:130-49. 